\title{
Analyse de la variabilité morphologique et enzymatique des formes cultivées et spontanées de Hedysarum coronarium L. en Tunisie
}

\author{
N. Trifi-Farah 1, W.S. Chatti 2 , M. Marrakchi 2 et J. Pernèst 3 \\ 1 Ecole Nationale des Ingénieurs de Sfax, BPW. 3038, Sfax; \\ 2 Faculté des Sciences de Tunis, laboratoire de génétique, Campus universitaire, 1060, Tunis, Tunisie; \\ 3 CNRS, Laboratoire de génétique et physiologie du développement des plantes, 91190 Gif-sur-Yvette, France
}

(reçu le 4 mai 1988, accepté le 15 mars 1989)

\begin{abstract}
Résumé - La variabilité morphologique et le polymorphisme enzymatique chez 3 types de populations de Hedysarum coronarium ont été étudiés grâce à une analyse en composantes principales et des paramètres de génétique des populations. II en ressort que les formes cultivées ont la même diversité génétique que les populations spontanées et ne se distinguent de celles-ci que par leurs caractères morphologiques. Les populations "synthétiques" se différencient des autres par leur faible diversité génétique, cependant elles se présentent comme des formes intermédiaires améliorées par rapport aux spontanées, sans pour autant atteindre la bonne vigueur des cultivars.
\end{abstract}

Hedysarum coronarium - polymorphisme enzymatique et morphologique

Summary - Morphological and enzymatic variability analysis of cultivated and spontaneous forms or Hedysarum coronarium. In the present study, the morphological variability and the enzymatic polymorphism in 3 population types in Hedysarum coronarium are described. Some parameters of population genetics and a principal component analysis were used in the study. The following results were found: cultivars and spontaneous populations present the same degree of genetic diversity but are very different as regards morphological characteristics. The artificial populations may be distinguished from the others by their very limited genetic diversity. However, they seem to be improved intermediate forms compared to the spontaneous populations; however, they are not as vigourous as the cultivars.

Hedysarum coronarium - enzymatic and morphological polymorphism

\section{Introduction}

L'Hedysarum coronarium ( $2 \mathrm{n}=16$ ), légumineuse connue sous le nom vulgaire de sulla ou sainfoin d'Espagne, est une espèce fourragère. Dans les endroits où le sulla pousse spontanément, les plantes, malgré des caractéristiques agronomiques limitées (port rampant, rendement fourrager faible...), sont exploitées par les agriculteurs tant pour la pâture que pour la protection des sols, particulièrement sur les pentes marneuses sensibles à l'érosion.

Par ailleurs, cette espèce est cultivée sporadiquement au nord de la Tunisie, notamment dans les régions de Béja, Mateur et Ain Draham, occupant ainsi quelques milliers d'hectares. Ces cultures, utilisant des variétés améliorées d'origine italienne, sont soit à vocation fourragère (pour l'affouragement en vert, pour l'ensilage ou pour le foin), soit destinées à la production de graines. Les rendements par hectare peuvent atteindre jusqu'à 90 tonnes de fourrage vert et 5 quintaux de graines (Le Houerou, 1965).

Ces variétés peuvent être semées, soit à l'état de gousses, soit à l'état de graines décortiquées. En Tunisie, les semis s'échelonnent du début du mois de septembre jusqu'à la fin octobre. Ils sont effectués à $2 \mathrm{~cm}$ de profondeur, en lignes écartées de $80 \mathrm{~cm}$ ou de 30 à $60 \mathrm{~cm}$ respectivement en culture pour la graine ou pour le fourrage.

Parmi les cultivars introduits en Tunisie, on peut citer :

- la variété Scaravatti 1187, qui a été importée d'Italie vers 1954 et qui fut longtemps cultivée en Tunisie; 
- Sparacia, d'origine sicilienne, introduite en Tunisie dans les années 1976-1977;

- Grimaldi, qui est actueliement la plus commercialisée en Tunisie.

Ces cultivars présentent des caractéristiques fourragères comparables à celles de la luzerne et du trèfle violet, en particulier les teneurs en matière sèche, matière azotée digestible... (Cenni et al., 1968; Ballatore, 1972).

Cependant, ces variétés cultivées en Tunisie sont mal définies. En effet, nous manquons d'indications précises quant à leur généalogie. De même, l'importation de plusieurs variétés, leur culture dans la même aire de distribution que les populations spontanées, ainsi que le régime préférentiellement allogame, facilitent le brassage génique entre les différentes formes spontanées et cultivées.

Dans ce travail, nous avons étudié les polymorphismes morphologiques et enzymatiques des formes naturelles spontanées prospectées en Tunisie, ainsi que des variétés cultivées. $P a r$ cette analyse, nous pensons contribuer à l'obtention de nouvelles formes améliorées à partir des populations spontanées locales, en les situant par rapport aux 2 formes décrites précédemment.

\section{Matériel et Méthodes}

\section{Matériel biologique}

Le matériel végétal utilisé provient de 3 origines :

- 9 populations spontanées prospectées à travers la Tunisie : les caractéristiques de ces populations sont mentionnées dans le Tableau I;

- 5 cultivars d'origine italienne cultivés en Tunisie dans les régions de Béja (BEC), Testour (TEC), Nefza (NEC), Chaouat (CHC) et Mateur (MAC) (Trifi-Farah et al., 1989);

Tableau I. Caractéristiques des stations d'origine des populations spontanées.

$\begin{array}{ll}\begin{array}{l}\text { Origine } \\ \text { géographique }\end{array} & \begin{array}{l}\text { Nomen- Latitude Longitude* } \\ \text { clature }\end{array}\end{array}$

\begin{tabular}{|c|c|c|c|}
\hline Béja & BES & $36^{\circ} 44^{\prime}$ & $9^{\circ} 1^{\prime}$ \\
\hline El Haouaria & EHS & $37^{\circ} \quad 3^{\prime}$ & $11^{\circ} 2^{\prime}$ \\
\hline Djebel Zit & ZTS & $36^{\circ} 30^{\prime}$ & $10^{\circ} 18^{\prime}$ \\
\hline Forêt Ain Djemala & FAS & $36^{\circ} 31^{\prime}$ & $9^{\circ} 28^{\prime}$ \\
\hline Tunis & TUS & $36^{\circ} 35^{\prime}$ & $10^{\circ} 8^{\prime}$ \\
\hline Kef & KFS & $36^{\circ} 1^{\prime}$ & $8^{\circ} 43^{\prime}$ \\
\hline Nacherine & NCS & $37^{\circ}$ & $10^{\circ} 4^{\prime}$ \\
\hline El Alia & EAS & $37^{\circ} 10^{\prime}$ & $10^{\circ} 6^{\prime}$ \\
\hline Kélibia & KES & $36^{\circ} 53^{\prime}$ & $11^{\circ} 5^{\prime}$ \\
\hline
\end{tabular}

- A l'est du méridien international.
- 4 populations $Z E_{1}, E Z_{1}, Z E_{3}$ et $Z E_{4}$ appelées «synthétiques", comme décrit par Trifi-Farah et al. (1989). Ces populations issues d'autofécondations, ont comme origine un croisement entre les individus EHS et ZTS.

\section{Méthodes d'analyse de la variabilité morpholo- gique}

Cette étude a été faite sur les caractères suivants : date d'apparition des première, deuxième et troisième feuilles $\left(D F_{1}, D F_{2}\right.$ et $D_{3}$ ), nombre de folioles des première, deuxième et troisième feuilles $\left(\mathrm{fF}_{1}, \mathrm{fF}_{2}\right.$ et $\left.\mathrm{fF}_{3}\right)$, longueur de l'axe orthotrope (LO), nombre de rameaux plagiotropes (NP), longueur du plus grand rameau plagiotrope (LP), longueur totale des axes aériens (LT), nombre moyen de folioles des 4 dernières feuilles de l'axe principal orthotrope (fO) (Fig. 1).

Les différents caractères ont été mesurés sur des individus appartenant aux populations suivantes : TUS, CHC, TEC, ZE $, E_{1}, Z_{1}, Z_{3}, Z E_{4}, K F S, N C S, E A S$ et KES. Chaque population est composée d'un échantillon comprenant de 20 à 46 individus.

Les résultats, exprimés en moyenne par population, sont ensuite soumis à une analyse en composantes principales (ANCOP); celle-ci est une étape permettant la description phénotypique des populations en mesurant les corrélations qui existent entre les moyennes de chacune d'entre elles.

\section{Méthodes d'analyse du polymorphisme enzyma- tique}

Le protocole expérimental, les systèmes enzymatiques étudiés ainsi que les données génétiques sur ces enzymes sont décrits par Trifi-Farah (1986).

Nous avons considéré, dans cette analyse, les systèmes enzymatiques suivants : alcool déshydrogénase $(A D H)$, malate déshydrogénase $(M D H)$, isocitrate déshydrogénase (ICD), phosphogluconate déshydrogénase (6-PGD), peroxydase (PER), glutamate oxaloacétate transaminase (GOT), phosphoglucomutase (PGM), estérases (EST), phosphatases acides (PHO) et phosphogluco-isomérase (PGI).

Concernant les méthodes d'analyse des résultats, nous avons utilisé 3 types de paramètres.

Les paramètres décrivant la structure intrapopulation en se basant sur le taux de polymorphisme (Cavalli-Sforza et Bodmer, 1971), le nombre moyen d'allèles par locus et la diversité génétique de $\mathrm{Nei}$ (Nei, 1972). Ce dernier paramètre représente la probabilité pour qu'un individu pris au hasard dans la population soit hétérozygote au locus $k$, sa formulation est :

$$
h_{k}=1-\sum_{i=1}^{1} x_{i}^{2} \quad x_{i}=\text { fréquence du } i^{\theta} \text { allèle à ce locus }
$$

Si I locus sont considérés, l'indice de diversité moyen sera calculé ainsi :

$$
\overline{\mathrm{h}}=\frac{1}{1} \sum_{\mathrm{k}=1}^{1} \mathrm{~h}_{\mathrm{k}}
$$

Les paramètres décrivant la structure propre des populations, en l'occurrence :

- un test $\chi^{2}$ de conformité calculé avec les fréquences génotypiques;

- un test $\chi^{2}$ avec "Pooling", dans le cas où il y a plus de 2 allèles par locus. Les différents génotypes sont 

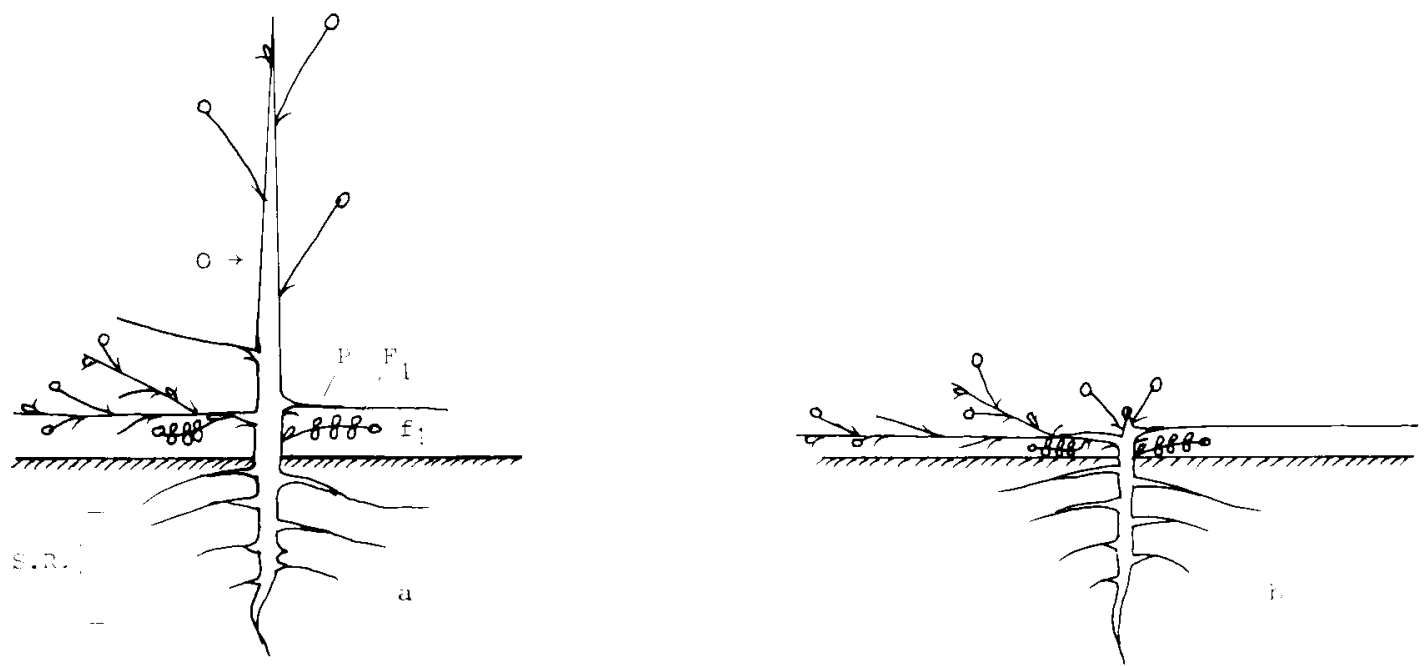

Fig. 1. Représentation schématique d'un pied fleuri d'H. coronarium. a . A axe principal orthotrope développé. b. A axe principal orthotrope non développé. $\mathrm{O}:$ axe principal orthotrope; $\mathrm{P}:$ axe latéral plagiotrope; $\mathrm{F}:$ feuille plurifoliolée; $f:$ foliole; $S R:$ système racinaire.

regroupés en 3 classes : les homozygotes pour l'allèle le plus fréquent, les hétérozygotes pour l'allèle le plus fréquent avec tous les autres allèles, enfin tous les autres génotypes (Sokal et Rohlf, 1969);

- un test non paramétrique (Elston et Forthofer, 1977) analogue au test d'exacte probabilité de Fischer pour un tableau de contingerice $2 \times 2$;

- un coefficient d'excès ou de défaut d'hétérozygotie défini comme suit :

$$
F=1-\frac{\text { hétérozygotie observée }}{\text { hétérozygotie attendue }}
$$

Les paramètres de diversité interpopulation, qui comportent :

- les paramètres de subdivision de Wright (Wright, 1978) permettant de calculer les variances des fréquences de l'allèle i $\left(q_{x(i)}\right)$, par rapport à la moyenne des fréquences de cet allèle $\left(q_{(i)}\right)$. Pour $K$ allèles et $S$ subdivisions, la formulation génerale est la suivante :

$$
F_{S T}=\sum_{i=1}^{k} \frac{1}{S} \sum_{x=1}^{S}\left(q x_{(i)}-\bar{q}_{(i)}\right)^{2}
$$

Pour un ensemble $T$ composé de $S$ subdivisions regroupant chacune $D$ populations, la différenciation des populations à l'intérieur des subdivisions $\left(F_{D S}\right)$ et la différenciation des populations par rapport au total $\left(F_{D T}\right)$ sont déterminées par la relation suivante :

$$
\left(1-F_{D T}\right)=\left(1-F_{S T}\right)\left(1-F_{D S}\right)
$$

Les distances génétiques dont celle non biaisée de Nei (1978) :

$$
\hat{D}=-\log \frac{\overline{G_{x y}}}{\sqrt{\overline{G_{x}} \overline{G_{y}}}}
$$

$\overline{G_{x}}$ et $\bar{G}_{y}$ sont respectivement les transformations non biaisées de $J_{x}$ et $J_{y}$.

$$
\begin{gathered}
J_{x y}=\sum_{i=1}^{k} q x_{(i)} q y_{(i)} \\
J_{x}=\sum_{i=1}^{k} q x_{(i)}^{2}
\end{gathered}
$$

$$
J_{y}=\sum_{i=1}^{k} q y_{(i)^{2}}
$$

$\mathrm{qx}_{(i)}$ et $q \mathrm{y}_{(i)}$ étant les fréquences respectives de l'allèle dans les populations $x$ et $y$.

Par ailleurs, nous avons fait également une analyse en composantes principales en utilisant simultanément les données morphologiques (moyenne par population) et enzymatiques (fréquences alléliques) obtenues à partir des populations TUS, KFS, NCS, EAS, KES, $\mathrm{TEC}, \mathrm{CHC}, \mathrm{EZ}_{1}$ et $\mathrm{ZE}_{1}$.

\section{Résultats}

\section{Analyse de la variabilité morphologique}

L'analyse en composantes principales présentée dans le Tableau II donne une estimation du pourcentage de variabilité représenté par chaque axe.

Nous nous sommes limités aux 3 premiers axes pour décrire la variabilité totale des populations. En effet, ces axes représentent à eux seuls $88 \%$ de la variabilité totale. La signification de ces composantes serait la suivante :

- La première décrit le développement végétatif général. Cette composante est définie du côté positif par la date d'apparition des troisièmes feuilles, indiçuant que les populations projetées de ce côté de l'axe forment tardivement les troisièmes feuilles. Du côté négatif, l'axe est défini par les caractères NP, LP et LT. En définitive, les populations projetées du côté positif semblent avoir un appareil végétatif à prédominance orthotropique et une apparition tardive des troisièmes feuilles.

- Le deuxième axe serait caractéristique des feuilles. II opposera les plantes qui forment pré- 
Tableau II. Analyse en composantes principales de l'étude morphologique.

\begin{tabular}{lcccc}
\hline $\begin{array}{l}\text { Composantes principales (axes) } \\
\text { Pourcentage d'inertie }\end{array}$ & 1 & 2 & 3 & 4 \\
\hline Variables contribuant & 60,73 & 20,26 & 7,79 & 6,73 \\
à la définition de l'axe & $\mathrm{NP}(-946)$ & $\mathrm{LO}(-428)$ & $\mathrm{LO}(+464)$ & $\mathrm{fF}(+689)$ \\
& $\mathrm{LP}(-980)$ & $\mathrm{fF}(+790)$ & $\mathrm{fF}_{3}(+377)$ & $\mathrm{fF}_{1}(+517)$ \\
& $\mathrm{DF}(+959)$ & $\mathrm{fO}(-963)$ & & $\mathrm{DF}_{2}(+432)$ \\
& $\mathrm{LT}(-917)$ & & &
\end{tabular}

+ ou - : corrélations positives ou négatives; NP : nombre de rameaux plagiotropes; LP : longueur du plus grand rameau plagiotrope; LT : longueur totale des axes aériens orthotropes et plagiotropes; $L O:$ longueur de l'axe orthotrope; fO : nombre moyen de folioles des 4 dernieres feuilles de l'axe principal orthotrope; $\mathrm{fF}_{1}, \mathrm{fF}_{2}, \mathrm{fF}_{3}$ : nombre de folioles des première, deuxième et troisième feuilles; $\mathrm{DF}_{1} ; \mathrm{DF}_{2}, \mathrm{DF}_{3}: \mathrm{date}_{\mathrm{d}}$ d'apparition des première, deuxième et troisième feuilles.

cocement les feuilles et qui présentent, à maturité, peu de folioles, aux plantes développant tardivement les feuilles et présentant à maturité beaucoup de folioles.

- Le troisième axe semble être lié à l'architecture globale de la plante, en rapport avec le développement de l'axe orthotrope.

En tenant compte de la signification de ces axes, nous avons analysé la dispersion des populations dans les plans engendrés par ces composantes.

L'analyse de la Figure 2, qui représente la dispersion des populations dans le plan des composantes 1 et 2, montre que :

- par rapport à l'axe 1, il y a opposition des populations spontanées aux cultivars, qui se caractérisent respectivement par un bon développement plagiotropique et par un important rameau orthotrope. Les populations "synthétiques" sont assez proches des formes cultivées;

- le deuxième axe oppose les populations cultivées à la population "synthétique» $\mathrm{ZE}_{4}$. Celle-ci forme précocement des feuilles et présente à

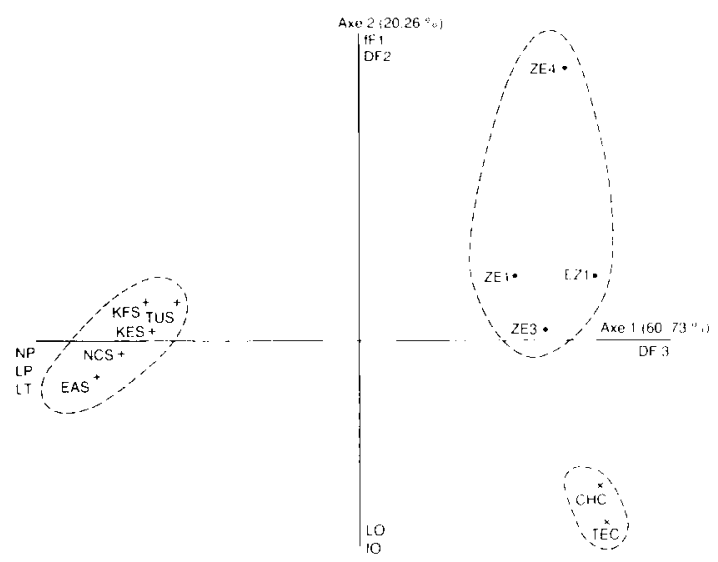

Fig. 2. Dispersion des populations dans le plan engendré par les 2 premiers axes de composantes principales. ${ }^{\circ}$ : population synthétique; ${ }^{x}$ : population cultivée; ${ }^{+}$: population spontanée. maturité peu de folioles. Les populations spontanées et les familles $Z E_{1}, E Z_{1}, Z E_{3}$ présentent une position intermédiaire.

La Figure 3, reflète la dispersion des populations selon la projection des axes 2 et 3 . Les résultats sont analogues à ceux issus de l'analyse de la Figure 2. Toutefois, on remarque que $\mathrm{ZE}_{4}$ est très différente des autres populations "synthétiques", particulièrement pour le développement de l'axe orthotrope. Les croisements entre populations bien distinctes suivis d'autofécondation permettent donc de générer des familles très différentes entre elles.

\section{Analyse de la variabilité enzymatique}

L'ensemble des données génétiques sur les systèmes enzymatiques nous a permis de détecter 16 locus dont les fréquences alléliques ont été déterminées. Nous avons utilisé ces fréquences pour étudier le polymorphisme enzymatique des diverses populations par les différents paramètres cités précédemment.

\section{Variabilité intrapopulation}

L'analyse des données du Tableau III, qui résume les résultats obtenus pour les différents paramètres décrivant la structure intrapopulation, montre :

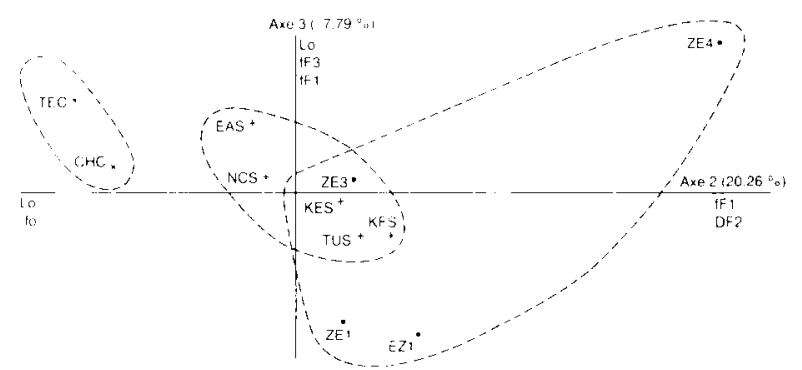

Fig. 3. Dispersion des populations dans le plan engendré par les axes de composantes principales 2 et $3 .^{\circ}$ : population synthétique; ${ }^{x}$ : population cultivée; ${ }^{+}$: population spontanée. 
Tableau III. Variabilité génétique étudiée aux 16 locus (erreurs standard).

\begin{tabular}{|c|c|c|c|c|}
\hline Populations & $\begin{array}{c}\text { Nombre } \\
\text { d'individus }\end{array}$ & $\begin{array}{l}\text { Pourcentage de } \\
\text { locus polymorphes } \\
\text { (seuil de } 5 \% \text { ) }\end{array}$ & $\begin{array}{l}\text { Nombre moyen } \\
\text { d'allèles par locus }\end{array}$ & $\begin{array}{l}\text { Indice de diversité } \\
\text { de Nei }\end{array}$ \\
\hline \multicolumn{5}{|c|}{ Populations cultivées } \\
\hline $\mathrm{CHC}$ & $\begin{array}{l}22 \\
(0)\end{array}$ & 18,8 & $\begin{array}{c}1,56 \\
(0,16)\end{array}$ & $\begin{array}{c}0,107 \\
(0,043)\end{array}$ \\
\hline BEC & $\begin{array}{l}22 \\
(0)\end{array}$ & 31,3 & $\begin{array}{c}1,44 \\
(0,13)\end{array}$ & $\begin{array}{c}0,123 \\
(0,046)\end{array}$ \\
\hline MAC & $\begin{array}{l}22 \\
(0)\end{array}$ & 25 & $\begin{array}{c}1,38 \\
(0,15)\end{array}$ & $\begin{array}{c}0,108 \\
(0,047)\end{array}$ \\
\hline TEC & $\begin{array}{l}24 \\
(0)\end{array}$ & 31,3 & $\begin{array}{c}1,56 \\
(0,16)\end{array}$ & $\begin{array}{c}0,140 \\
(0,051)\end{array}$ \\
\hline NEC & $\begin{array}{c}24 \\
(0)\end{array}$ & 37,5 & $\begin{array}{c}1,50 \\
(0,18)\end{array}$ & $\begin{array}{c}0,151 \\
(0,054)\end{array}$ \\
\hline \multicolumn{5}{|c|}{ Populations synthétiques } \\
\hline ZE1 & $\begin{array}{l}32 \\
(0)\end{array}$ & 31,3 & $\begin{array}{c}1,38 \\
(0,15)\end{array}$ & $\begin{array}{c}0,093 \\
(0,043)\end{array}$ \\
\hline EZ1 & $\begin{array}{c}31,3 \\
(0,1)\end{array}$ & 25 & $\begin{array}{c}1,31 \\
(0,15)\end{array}$ & $\begin{array}{c}0,111 \\
(0,050)\end{array}$ \\
\hline \multicolumn{5}{|c|}{ Populations spontanées } \\
\hline FAS & $\begin{array}{l}24 \\
(0)\end{array}$ & 37,5 & $\begin{array}{c}1,50 \\
(0,16)\end{array}$ & $\begin{array}{c}0,139 \\
(0,050)\end{array}$ \\
\hline EHS & $\begin{array}{l}24 \\
(0)\end{array}$ & 31,3 & $\begin{array}{c}1,38 \\
(0,15)\end{array}$ & $\begin{array}{c}0,131 \\
(0,055)\end{array}$ \\
\hline ZTS & $\begin{array}{l}22 \\
(0)\end{array}$ & 31,3 & $\begin{array}{c}1,44 \\
(0,13)\end{array}$ & $\begin{array}{c}0,153 \\
(0,055)\end{array}$ \\
\hline BES & $\begin{array}{l}23 \\
(0)\end{array}$ & 31,3 & $\begin{array}{l}1,44 \\
(0,16)\end{array}$ & $\begin{array}{l}0,114 \\
(0,045)\end{array}$ \\
\hline KFS & $\begin{array}{l}22 \\
(0)\end{array}$ & 31,3 & $\begin{array}{c}1,38 \\
(0,15)\end{array}$ & $\begin{array}{c}0,121 \\
(0,048)\end{array}$ \\
\hline NCS & $\begin{array}{c}22,9 \\
(0,1)\end{array}$ & 43,8 & $\begin{array}{c}1,63 \\
(0,18)\end{array}$ & $\begin{array}{c}0,195 \\
(0,059)\end{array}$ \\
\hline TUS & $\begin{array}{l}23 \\
(0)\end{array}$ & 43,8 & $\begin{array}{c}1,63 \\
(0,15)\end{array}$ & $\begin{array}{c}0,159 \\
(0,049)\end{array}$ \\
\hline EAS & $\begin{array}{l}23 \\
(0)\end{array}$ & 31,3 & $\begin{array}{c}1,38 \\
(0,15)\end{array}$ & $\begin{array}{c}0,112 \\
(0,045)\end{array}$ \\
\hline KES & $\begin{array}{l}24 \\
(0)\end{array}$ & 43,8 & $\begin{array}{c}1,50 \\
(0,13)\end{array}$ & $\begin{array}{c}0,171 \\
(0,052)\end{array}$ \\
\hline
\end{tabular}

- comme prévu, les populations spontanées sont relativement plus polymorphes que les cultivées. En effet, le taux de polymorphisme varie de 31,3 à 43,8 chez les populations spontanées, alors qu'il varie de 18,8 à 37,5 chez les cultivées. Les populations "synthétiques" seraient également moins polymorphes que les spontanées;

- les résultats montrent que la valeur du nombre moyen d'allèles par locus, est de 1,46. Les populations "synthétiques" ayant un nombre moyen d'allèles par locus égal à 1,35 semblent être les moins variables;

- en tenant compte de la diversité génétique de $\mathrm{Nei}$, on peut dire que la variabilité des formes spontanées est légèrement plus importante que celle des cultivées. La variabilité est la plus faible chez les familles "synthétiques» $(h=0,102)$.

\section{Structure propre des populations}

L'utilisation des paramètres décrivant cette structure suppose connue pour chaque locus la composition génotypique des populations. En conséquence, nous avons effectué les différents tests uniquement à partir de 6 locus (PGMA, PGIA, PGDB, ADHA, ICDA et GOTB). L'écart à la panmixie a été estimé en utilisant les différents paramètres décrits dans "Matériel et Méthodes". Les résultats ainsi obtenus sont résumés dans le Tableau IV. Ces résultats montrent que l'ensemble des populations sont dans un état d'équilibre panmictique. Toutefois, on note que parmi les 16 populations analysées, 10 d'entre elles se sont révélées non panmictiques pour le locus PGDB. Ce résultat s'expliquerait par une sélection de l'un des 2 allèles de ce gène, ayant 
Tableau IV. Etude de la structure propre des populations : écart à la panmixie.

\begin{tabular}{|c|c|c|c|c|c|c|}
\hline Populations & $P G M A$ & $P G I A$ & $\begin{array}{l}\text { Locus } \\
P G D B\end{array}$ & $A D H A$ & $I C D A$ & GOTB \\
\hline \multicolumn{7}{|l|}{ Cultivées } \\
\hline $\mathrm{CHC}$ & $M$ & $P$ & $\begin{array}{l}\text { NP } \\
(-0,913)\end{array}$ & $M$ & $P$ & $P$ \\
\hline BEC & $M$ & $P$ & $\mathrm{P}$ & M & M & $P$ \\
\hline MAC & $P$ & $P$ & $\begin{array}{l}\text { NP } \\
(-0,636)\end{array}$ & $\mathrm{M}$ & $M$ & $M$ \\
\hline TEC & $P$ & $P$ & $\begin{array}{l}\text { NP } \\
(-0,467)\end{array}$ & $M$ & $P$ & M \\
\hline NEC & M & $P$ & $\begin{array}{l}N P \\
(-0,846)\end{array}$ & $M$ & $P$ & $M$ \\
\hline \multicolumn{7}{|l|}{ Synthétiques } \\
\hline ZEI & M & $M$ & $P$ & M & M & M \\
\hline EZI & M & M & $\mathrm{P}$ & $M$ & $M$ & M \\
\hline \multicolumn{7}{|l|}{ Spontanées } \\
\hline FAS & $\mathrm{P}$ & $P$ & $P$ & $M$ & $M$ & M \\
\hline EHS & M & $P$ & $\begin{array}{l}\text { NP } \\
(-0,753)\end{array}$ & $M$ & $M$ & $M$ \\
\hline ZTS & M & $P$ & $\begin{array}{l}\text { NP } \\
(-0,731)\end{array}$ & $P$ & M & M \\
\hline BES & M & $P$ & $\begin{array}{l}\mathrm{NP} \\
(-0,742)\end{array}$ & $M$ & $P$ & M \\
\hline KFS & M & $P$ & $\begin{array}{l}\text { NP } \\
(+0,654)\end{array}$ & $M$ & M & M \\
\hline NCS & $P$ & $P$ & $\begin{array}{l}\text { NP } \\
(+0,607)\end{array}$ & $M$ & M & M \\
\hline TUS & $\mathrm{P}$ & $P$ & $\mathrm{P}$ & $M$ & M & M \\
\hline EAS & M & $P$ & $\begin{array}{l}\mathrm{NP} \\
(-0,667)\end{array}$ & $M$ & M & M \\
\hline KES & $\begin{array}{l}N P \\
(-0,652)\end{array}$ & $P$ & $\begin{array}{l}\text { NP } \\
(-0,652)\end{array}$ & $M$ & $M$ & $P$ \\
\hline
\end{tabular}

$P$ : locus panmictique; $M$ : locus monomorphe; NP : locus en écart à la panmixie avec excès $(+)$ ou défaut $(-)$ d'hétérozygotes.

comme effet de diminuer simultanément les taux des hétérozygotes et de l'un des homozygotes. La raison essentielle de cette sélection n'est pas encore bien connue.

\section{Variabilité interpopulation}

Cette étude peut être relatée de 3 manières.

Du point de vue du facteur de subdivision de Wright, on observe une grande hétérogénéité entre les différentes populations étudiées ( $F_{D T}$ moyen $=0,365)$. L'hétérogénéité interpopulation $\left(F_{\mathrm{ST}}=0,102\right)$ est supérieure à l'hétérogénéité intrapopulation ( $\left.F_{D S}=0,003\right)$.

En outre, parmi les locus analysés, certains entraînent un maximum de variabilité (GOTC, GOTA), tandis que les locus PGMA, ICDA et GOTB contribuent le moins à cette variabilité.

En tenant compte de la distance de Nei non biaisée, les résultats obtenus et résumés dans la Figure 4 montrent, en particulier, un regroupement des familles "synthétiques» $Z E_{1}$ et $E Z_{1}$.
Les formes cultivées et spontanées, caractérisées par une grande hétérogénéité, ne constituent pas de groupe particulier et ne semblent pas se rapprocher selon leur origine géographique.

Analyse en composantes principales des données morphologiques et enzymatiques

Faisant suite à l'analyse séparée de la variabilité morphologique et enzymatique, nous avons réali-

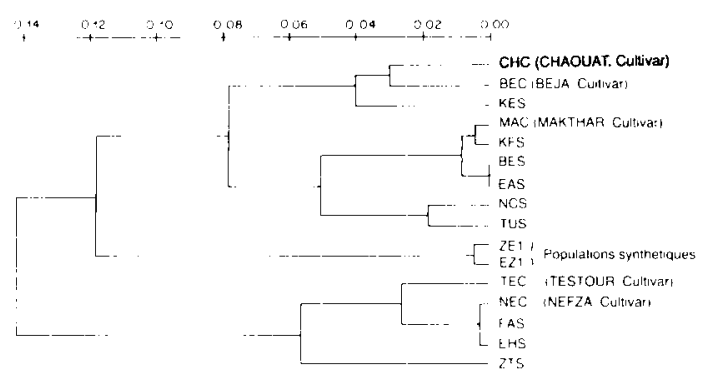

Fig. 4. Distance de Nei non biaisée. 
sé une analyse en composantes principales utilisant l'ensemble des données morphologiques et enzymatiques. Cette étude permettrait de mieux estimer le rapport entre la domestication des familles synthétiques et la vigueur des plantes.

Cette étude a été faite sur les populations $E Z_{1}$ et $Z E_{1}$ ("synthétiques»), CHC et TEC (cultivées) et TUS, KFS, NCS, EAS et KES (spontanées).

Cette analyse en composantes principales nous a permis de définir les différentes composantes et d'estimer le pourcentage de variabilité qu'elles représentent (Tableau V).

Tableau V. Définition des axes de l'ACP étudiés sur l'ensemble des variables.

\begin{tabular}{|c|c|c|c|c|}
\hline Axe & 1 & 2 & 3 & 4 \\
\hline $\begin{array}{l}\text { Pourcentage } \\
\text { d'inertie }\end{array}$ & 39,06 & 27,33 & 12,74 & 8,80 \\
\hline Variables & $\begin{array}{l}{ }^{*} \text { FF3 } \\
(-963) \\
* \text { LT } \\
(-894) \\
. \text { PGMB } \\
(+867) \\
. \text { PGMC } \\
(-869) \\
. \text { ICDA } \\
(-868) \\
. \text { ICDB } \\
(+867) \\
. \text { GOTB } \\
(-896) \\
. \text { GOAA } \\
\text { (+896) }\end{array}$ & $\begin{array}{l}* \text { LO } \\
(-915) \\
* \text { NP } \\
(+855) \\
* \text { LP } \\
(+843) \\
* \text { FO } \\
(-857) \\
. \text { PGBA } \\
(-647) \\
. \text { PGBB } \\
(+647) \\
. \text { PGIA } \\
(+668)\end{array}$ & $\begin{array}{l}{ }^{*} \text { DF3 } \\
(+858) \\
. \text { GOAA } \\
(-636) \\
. \text { GOAA } \\
(+636) \\
. \text { ADAA } \\
(-607)\end{array}$ & $\begin{array}{l}\text { * DF1 } \\
(-787) \\
. \text { PGMA } \\
(+780) \\
. \text { PGIB } \\
(-739)\end{array}$ \\
\hline
\end{tabular}

" caracteres morphologiques; . variables enzymatiques; $(+)$ : corrélations positives; $(-)$ : corrélations négatives.

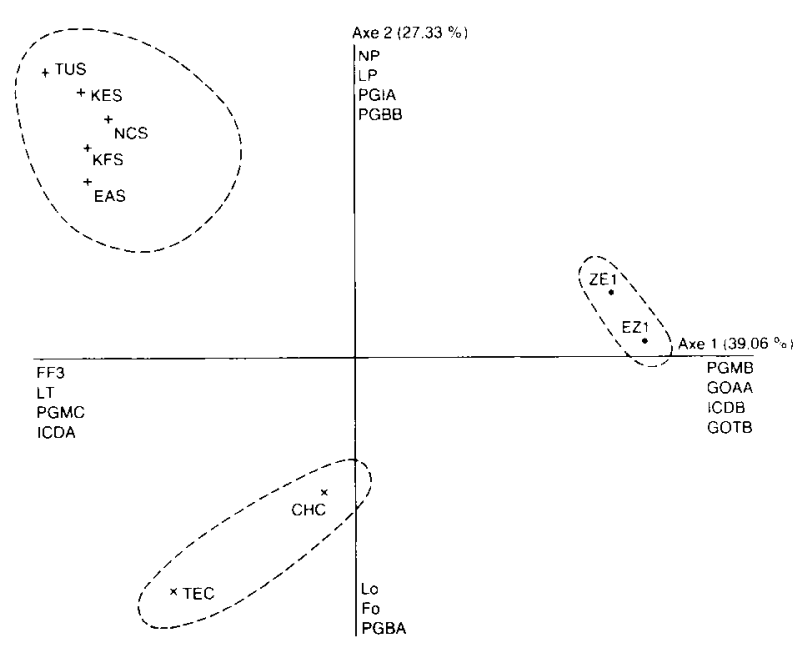

Fig. 5. Analyse en composantes principales de l'ensemble des caractères morphologiques et enzymatiques (plan des axes 1,2$){ }^{\circ}$ : population synthétique; $x$ : population cultivée; ${ }^{+}$ : population spontanée.
Les 4 premiers axes définissent $87,93 \%$ de la variabilité. II semble que les variables enzymatiques aient une grande importance dans la définition du premier axe. En revanche, les variables décrivant la morphologie sont considérées essentiellement dans la définition de la $2^{e} \mathrm{com}$ posante (axe 2), qui oppose ainsi les 2 tendances géotropiques.

Par ailleurs, la projection dans le plan des axes principaux représentée dans la Figure 5 indique, sur l'axe 1, une nette distinction entre les familles "synthétiques» et le reste des populations étudiées; selon l'axe 2, on observe aussi une très forte différence entre les populations spontanées et les formes cultivées. Ce même axe permet de placer les familles "synthétiques" dans une situation intermédiaire par rapport aux formes cultivées et spontanées.

Les familles $Z E_{1}$ et $E Z_{1}$, en étant fortement corrélées avec l'axe 1 , semblent se regrouper grâce à leurs caractères enzymatiques.

\section{Discussion et Conclusion}

Cette étude montre clairement que les caractères morphologiques permettent de différencier les formes spontanées locales de sulla, des cultivars d'origine italienne. En effet, ces 2 formes semblent se caractériser par des tendances géotropiques opposées : les cultivées sont érigées et peu ramifiées alors que les spontanées sont prostrées et très ramifiées. Des résultats analogues ont été obtenus par Bono (1973) et Marchais (1982) en analysant plusieurs variétés de mil pennisétaire cultivées en Afrique occidentale. En outre, les populations synthétiques se comportent comme des intermédiaires améliorés par le redressement de leur axe orthotrope, sans atteindre pour autant la vigueur des cultivars. Ainsi, on peut dire que la sélection de ces nouvelles formes semble emprunter le sens de la domestication.

L'analyse du polymorphisme enzymatique a mis en évidence la grande variabilité des formes cultivées et des formes spontanées. II est donc clair que les cultivars, bien qu'ils conservent leurs caractéristiques morphologiques et agronomiques, ne sont pas appauvris en diversité génétique. Des travaux similaires réalisés sur le mil pennisétaire par Tostain et al. (1987) ont montré que les formes sauvages sont moins variables que les cultivées. En revanche, les familles "synthétiques" se sont montrées les moins variables, traduisant ainsi la chute de vigueur due aux autofécondations réalisées au cours des sélections de ces formes en vue de leur domestication. En effet, il est généralement admis que la domesti- 
cation réduit la diversité génétique comme cela a été démontré chez le riz (Second, 1982).

Par ailleurs, en utilisant simultanément les données morphologiques et enzymatiques, l'analyse en composantes principales montre que les familles synthétiques, en se rapprochant des formes cultivées par leur morphologie, se différencient de l'ensemble des populations par leur faible variabilité enzymatique. Ce résultat s'expliquerait par la perte d'allèles due à un effet de dérive au moment du tirage des parents de la génération suivante, celle-ci n'ayant plus le même échantillonnage d'allèles que les populations spontanées EHS et ZTS dont elle est issue. Le taux d'hétérozygotes baisse également à cause des autofécondations réalisées pour aboutir à ces familles «synthétiques».

En outre, les résultats montrent que les formes spontanées et cultivées sont relativement proches du point de vue enzymatique. Ce rapprochement tire son explication, d'une part, du régime préférentiellement allogame de cette espèce, et d'autre part, de la proximité des peuplements spontanés et cultivés qui facilitent l'échange entre les formes. Nous pensons que la confrontation de nos résultats avec ceux obtenus par Damerval et al. (1987) chez le maïs, Tostain et al. (1987) chez le mil pourrait contribuer à la connaissance des processus évolutifs chez $H$. coronarium.

Quoi qu'il en soit, l'origine mal définie des cultivars d'origine italienne ne permet pas d'estimer les changements qui ont accompagné leur introduction en Tunisie.

Deux hypothèses pourraient être émises pour rendre compte de ces observations :

- les cultivars italiens ne sont pas à l'origine différents des populations naturelles de $\mathrm{H}$. coronarium rencontrées en Tunisie. Ainsi, ils s'y adaptent sans perdre leurs caractéristiques morphologiques;

- les cultivars italiens sont sélectionnés probablement sur des critères agronomiques et morphologiques. La variabilité enzymatique observée serait due à un brassage génique avec les populations locales spontanées.

Nos résultats ne permettent pas encore de choisir entre ces 2 hypothèses.

\section{Références}

Ballatore J.P. (1972) La produzione foraggera negli ambianti semiaridi, con particolare referimento alla Sicilia. Inf. Agrario 27, 7433-7464

Bono M. (1973) Contribution à la morphosystématique des Pennisetum annuels cultivés pour leur grain en Afrique occidentale francophone. Agron. Trop. 28, 229-356

Cavalli-Sforza L.L. \& Bodmer W.F. (1971) The Genetic of Human Populations. W.H. Freeman and Co., San Francisco

Cenni B., Jannella G. \& Colombani N. (1968) Chemical composition digestibility and nutritive value of sulla (Hedysarum coronarium L.) high produced in Voltera district. Ann. Fac. Med. Vet. Univ. Pisa 20, 155-168

Damerval C., Hebert Y. \& De Vienne D. (1987) Is the polymorphism of protein amounts related to phenotypic variability? A comparison of two dimensional electrophoresis data with morphological traits in maize. Theor. App. Genet. 74, 194-202

Elston R.C. \& Forthofer R. (1977) Testing for HardyWeinberg equilibrium in small samples. Biometrics 33 , 356-542

Le Houerou H.N. (1965) Les Cultures Fourragères en Tunisie. Documents techniques INRA n 13 , Tunisie

Marchais L. (1982) La diversité phénotypique des mils pennisétaires cultivés au Sénégal et au Mali. Agron. Trop. 37, 68-80

Nei M. (1972) Genetic distance between populations. Am. Nat. 106, 283-293

Nei M. (1978) Estimation of average heterozygosity and genetic distance from a small number of individuals. Genetics 89, 583-590

Second F. (1982) Origin of the genic diversity of cultivated rice (Oryza sp.) : study of the polymorphism scored at 40 isozyme loci. Jpn J. Genet. 57, 25-57

Sokal R.R. \& Rohlf F.J.(1969) Biometry W.H. Freeman and Co., San Francisco

Tostain S., Riandey M.F. \& Marchais L. (1987) Enzyme diversity in pearl millet (Pennisetum glaucum) in West Africa. Theor. Appl. Genet. 74, 188-193

Trifi-Farah N. (1986) Analyse de la variabilité morphologique et enzymatique : relations entre formes cultivées et spontanées de l' Hedysarum coronarium $L$. Thèse Sci., Tunis

Trifi-Farah N., Chatti W.S., Marrakchi M. et Pernès J. (1989) Déterminisme génétique de dix systèmes enzymatiques chez Hedysarum coronarium L. Agronomie, 9, 503-510

Wright S. (1978) Evolution and The Genetics of Populations. The University of Chicago Press, pp. 79-103 